\title{
ESTUDO EXPERIMENTAL E DE SIMULAÇÃO CFD-DEM DO ESCOAMENTO DE GRÃOS DE FERTILIZANTES EM UM DISCO GIRATÓRIO
}

\author{
D.D.L. COSTA ${ }^{1}$, D.L. SOUZA ${ }^{1}$, K.G. SANTOS ${ }^{1}$, J.L. VIEIRA NETO ${ }^{1 *}$ \\ ${ }^{1}$ Universidade Federal Do Triângulo Mineiro, Departamento de Engenharia Química \\ E-mail para contato: jose.neto@uftm.edu.br
}

\begin{abstract}
RESUMO - Na indústria de fertilizantes a granulação é uma operação essencial. $\mathrm{O}$ produto granulado tem várias propriedades aprimoradas e atende a requisitos de tamanho e forma. Este trabalho teve como objetivo analisar o comportamento de grãos de fertilizantes escoando em um disco rotativo. Assim, simulações foram realizadas através das técnicas de Dinâmica de Fluidos Computacionais (CFD) e Elementos Discretos (DEM), para avaliar parâmetros: coeficiente de restituição $(\eta)$, coeficiente de fricção $(\mu)$ e coeficiente de elasticidade $(k)$ do modelo linear spring-dashpot. Também foram avaliadas as variáveis, velocidade de rotação do eixo e grau de preenchimento, sobre os regimes experimentais de escoamento: rolamento, cascata e centrifugação. Além disso, verificou-se qual o ângulo de repouso dinâmico.
\end{abstract}

\section{INTRODUÇÃO}

Na produção de fertilizantes a granulação é uma etapa essencial para formar grânulos de boa qualidade. Este trabalho visa estudar a fluidodinâmica de grãos de fertilizantes em um granulador tipo disco rotativo avaliando algumas variáveis, tais como, rotação do eixo e grau de preenchimento, e observando a existência dos regimes: rolamento, cascata e centrifugação.

As técnicas de modelagem dependem da escolha de uma abordagem contínua Euleriana ou discreta Lagrangiana para as fases líquida e sólida. A primeira abordagem computacional Lagrange para partículas, denominada Modelo de Elementos Discretos (DEM), foi feita por Cundall e Strack (1979). A ideia básica do DEM é que a trajetória de cada partícula dentro do sistema é calculada, considerando todas as forças que atuam sobre ela e integrando a segunda lei de movimento de Newton e as equações cinemáticas para posição e orientação. Fluxos granulares foram reproduzidos com sucesso por simulações DEM de processos, incluindo leito de jorro (Bortolotti et al. 2013); leito empacotado (Garg et al. 2012) e discos rotativos (Silvério et al. 2014), dentre outros.

Di Renzo e Di Maio (2004) testaram a precisão de três diferentes modelos de força de contato, comparando os resultados, microscópicamente, com soluções analíticas quase exatas e, macroscopicamente, com resultados experimentais. Os resultados mostraram que o modelo spring-dashpot apresentou bons resultados para previsão de forças de contato. O termo "spring" refere-se à contribuição da resposta às forças elásticas enquanto o termo "dashpot" se refere à dissipação devido à deformação plástica. Assim, apenas colisões friccionais e elásticas são consideradas, avaliadas pelos seguintes parâmetros: coeficientes elásticos normais $\left(K_{n}\right)$ e tangenciais $\left(K_{t}\right)$, o coeficiente de fricção $\left(\mu_{f}\right)$ e o coeficiente de restituição $(\eta)$. 


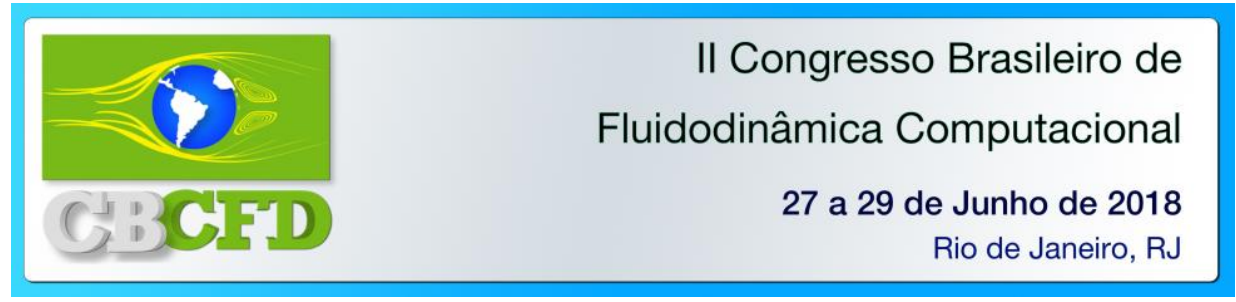

\section{MÉTODO}

\subsection{Metodologia Experimental}

A Figura 1 mostra o aparato utilizado no estudo de fluidodinâmica. O dispositivo foi instalado no Laboratório da UFTM.

(a)

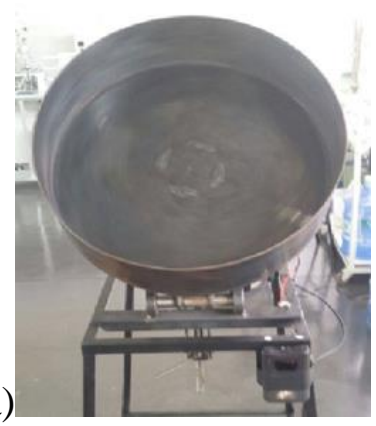

(b)

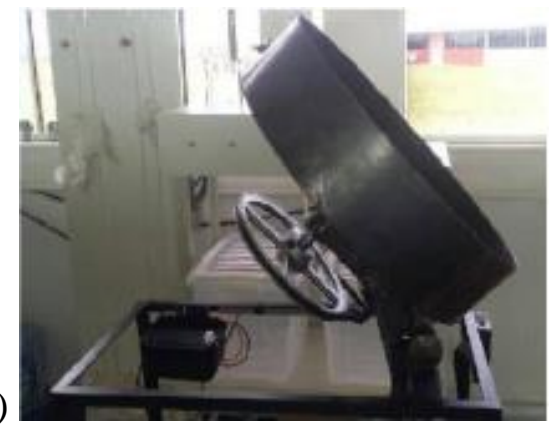

Figura 1 - Disco rotativo usado nos testes experimentais: (a) Vista frontal; (b) Vista lateral.

$\mathrm{O}$ disco rotativo tem $0,6 \mathrm{~m}$ de diâmetro e $0,06 \mathrm{~m}$ de altura, com um eixo rotativo acionado por um motor com $775 \mathrm{~W}, 90 \mathrm{~V}$ e rotação máxima de $4300 \mathrm{rpm}$. Uma conexão entre o motor e o disco foi feita por acoplamento a um redutor de velocidade, que controla a rotação e permite trabalhar dentro da faixa desejada para processos de granulação. As partículas neste estudo foram fertilizantes HERINGER, sob a forma de superfosfato único granular (GSS), com 2,99 mm de diâmetro médio Sauter e densidade de $1863 \mathrm{~kg} \cdot \mathrm{m}^{-3}$. Os testes fluidodinâmicos foram conduzidos com o disco inclinado a 45 graus.

\subsection{Metodologia de Simulação}

A malha computacional tridimensional com 41.398 células foi obtida a partir do software Gambit ${ }^{\circledR}$ e foi construída para garantir que o tamanho das células fosse pelo menos três vezes maior do que o diâmetro da esfera de tamanho de partícula igual $\left(\mathrm{d}_{\mathrm{P}}\right)$.

Neste trabalho, foi utilizada a equação governante do modelo Euleriano para simulação CFD da fase gasosa usando o pacote Fluent $\AA$. Uma vez resolvidas as equações de transporte para a fase contínua (fase gasosa), foi simulada a fase discreta (fase particulada) de acordo com uma abordagem Lagrangiana usando o Método de Elementos Discretos (DEM). ANSYS Fluent ${ }^{\circledR}$ permite usar o DEM como parte do modelo de fase discreta (DPM). O acoplamento entre as fases e seu impacto em ambos os caminhos da fase discreta e o fluxo de fase contínua está incluído nos cálculos. Por CFD-DEM calcula-se o campo de velocidade para a fase fluida e usa esses dados para calcular a fase particulada. Neste caso, utilizou-se o ar estagnado proposto, de modo que a interação do fluido de gás foi de pouca relevância, ou seja, foi usado DEM com uma ferramenta CFD. As equações dos modelos adotados neste trabalho foram descritas em estudos anteriores [6].

As seguintes condições foram utilizadas nas simulações: passo de tempo para a fase contínua 0.001; passo de tempo para a fase discreta 0,0001 e número de partículas injetadas 30.670 (para representar a massa de partículas de 803,5 g). As paredes foram consideradas uma condição limite de anti-deslizamento (no slip) e a rotação para cada regime foi definida por uma velocidade de rotação (rpm) na parede. Os resíduos foram de $10^{-3}$ e o tempo simulado variou entre 12 a 40 segundos para cada condição. $\mathrm{O}$ acoplamento entre velocidade e pressão definida pelo algoritmo SIMPLE. Para a discretização espacial foi empregada o modelo QUICK e a discretização temporal pelo modelo implícito FIRST ORDER. 


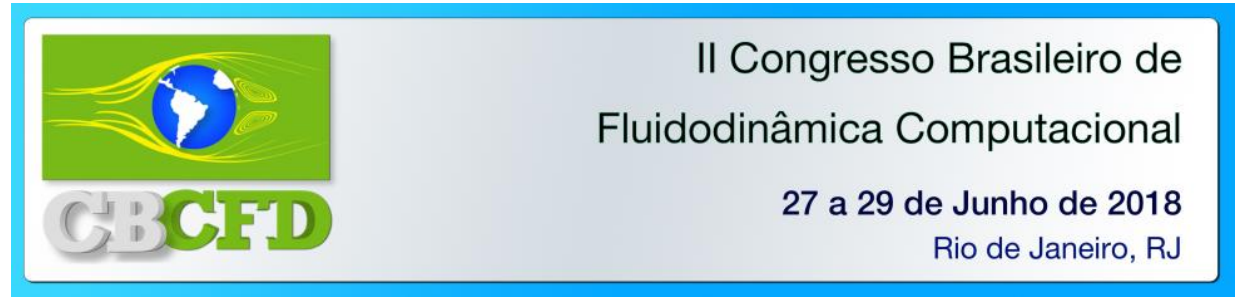

\section{RESULTADOS E DISCUSSÕES}

As Figuras 2 e 3 apresentam os regimes experimentais observados para dois graus de enchimento diferentes do disco rotativo com massa de fertilizantes: 803,5 g (Fig. 2) e $1.607 \mathrm{~g}$ (Fig. 3), respectivamente. Comparando as Figuras 2 e 3, verificou-se que para um grau de enchimento mais elevado (1607 g de fertilizantes), as partículas iniciaram os regimes de rolamento e cascata com velocidades de rotação ligeiramente menores, a 11 e $27 \mathrm{rpm}$, do que observado para o menor grau de enchimento (803,5 g de fertilizantes) a 13 e $34 \mathrm{rpm}$. Enquanto que para o regime de centrifugação não houve diferença, obtido com $51 \mathrm{rpm}$.

(a)

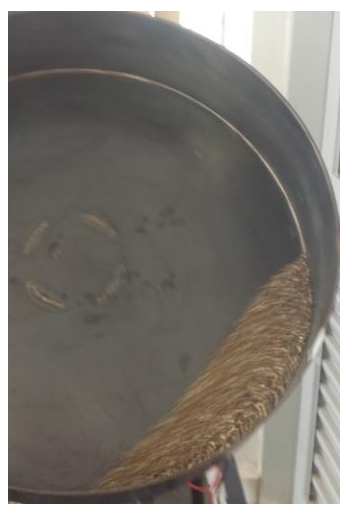

(b)

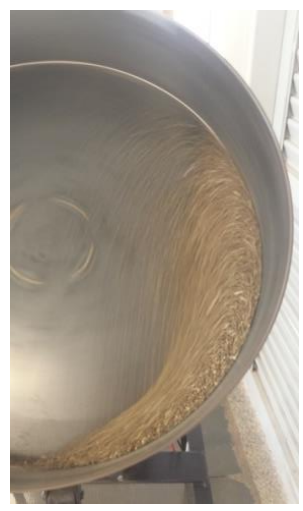

(c)

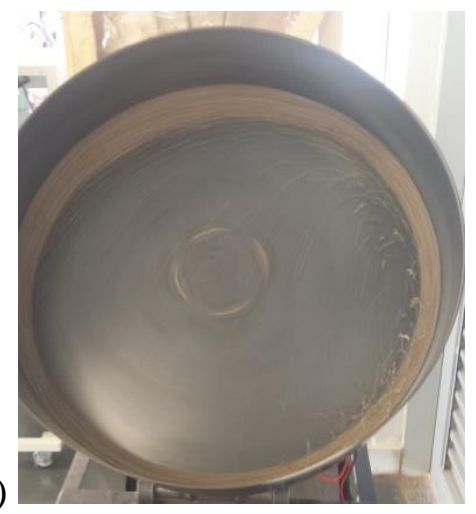

Figura 2 - Regimes no disco rotativo para o primeiro grau de enchimento, 803,5 g de fertilizantes: (a) rolamento a $13 \mathrm{rpm}$; (b) cascata a $34 \mathrm{rpm}$; (c) centrifugação a $51 \mathrm{rpm}$.

(a)

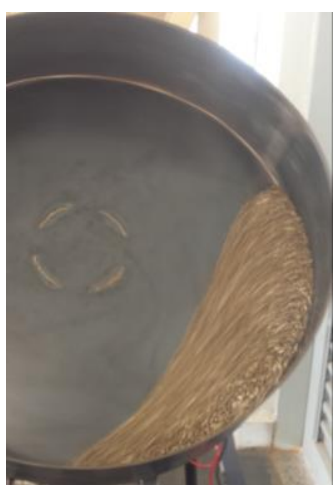

(b)

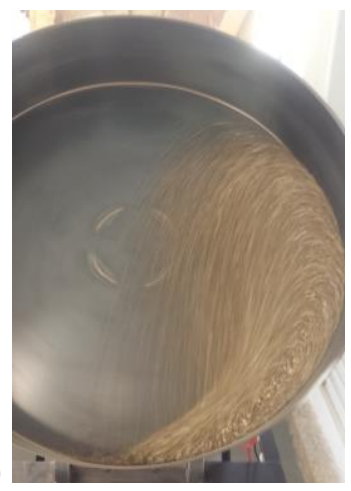

(c)

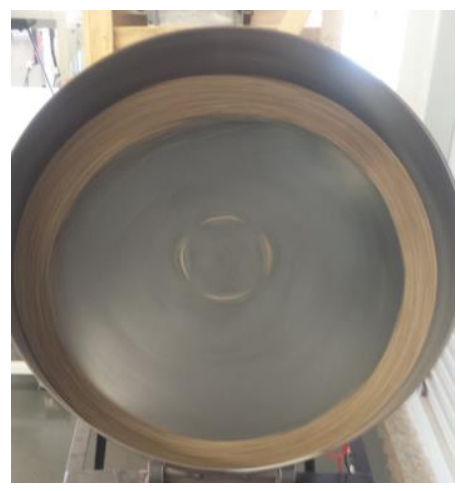

Figura 3 - Regimes no disco rotativo para o segundo grau de enchimento, $1.607 \mathrm{~g}$ de fertilizantes: (a) rolamento a $11 \mathrm{rpm}$; (b) cascata a $27 \mathrm{rpm}$; (c) centrifugação a $51 \mathrm{rpm}$.

Observou-se que os parâmetros mostrados na Fig. (4a) não levaram à centrifugação e as partículas ficaram posicionadas na parte inferior do disco. Na disposição da Fig. (4c) quase se obteve a condição de centrifugação, que ficou mais evidenciada na Fig. (4b) com os parâmetros: $k=400, \eta=0,5, \mu_{f}=0,5$.

Além disso, uma boa concordância foi encontrada na Figura 5 para o ângulo dinâmico experimental de repouso $\left(50^{\circ}\right)$ e o resultado simulado $\left(55^{\circ}\right)$ para melhor disposição dos parâmetros do modelo spring-dashpot $\left(k=400, \eta=0,5, \mu_{f}=0,5\right)$ com desvio de quase $10 \%$. Estes resultados foram obtidos na faixa de rolamento com $13 \mathrm{rpm}$ e menor massa de partículas $(803,5 \mathrm{~g})$. 


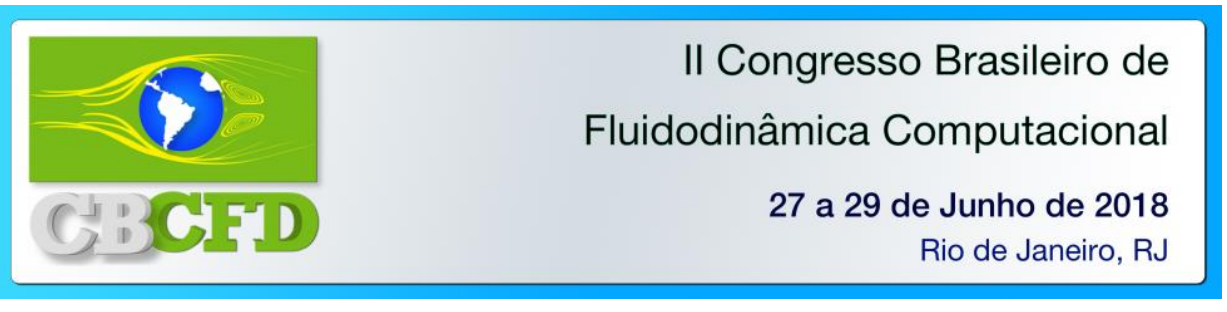

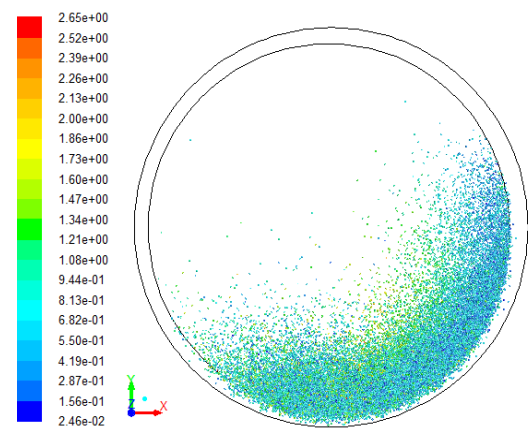

(a) $k=400, \eta=0.8, \mu_{f}=0.2$

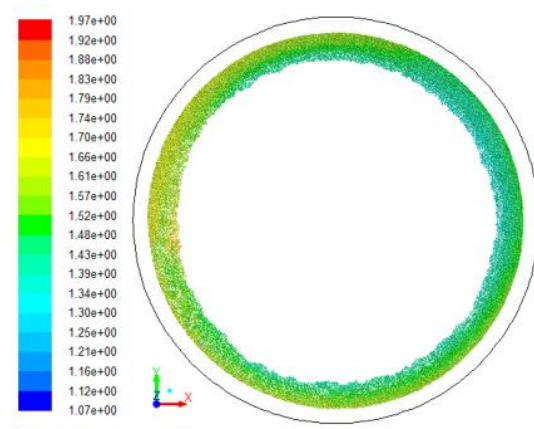

(b) $k=400, \eta=0.5, \mu_{f}=0.5$

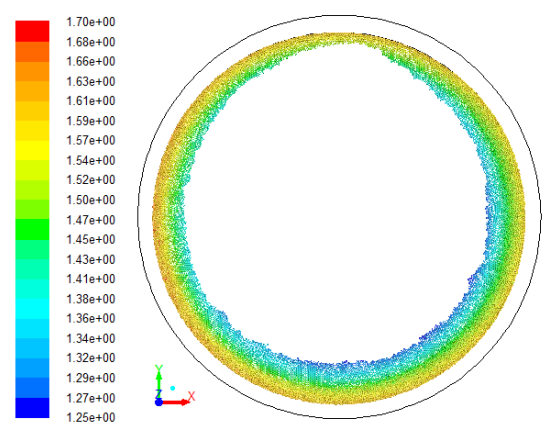

(c) $k=400, \eta=0.8 \mu_{f}=0.9$

Figura 4 - Condição centrifugação (51 rpm, 803,5 g), parâmetros do modelo spring-dashpot: (a) $k=400, \eta=0,8, \mu_{f}=0,2$; (b) $k=400, \eta=0,5, \mu_{f}=0,5$; (c) $k=400, \eta=0.8 \mu_{f}=0.9$.

(a)

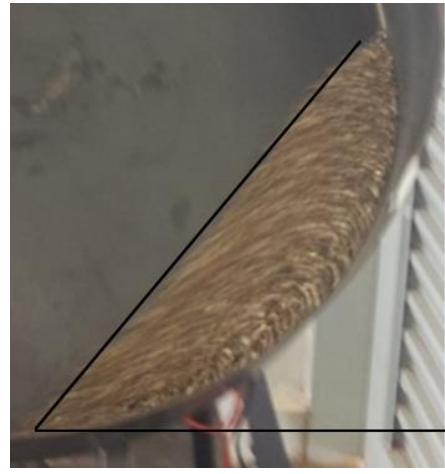

(b)

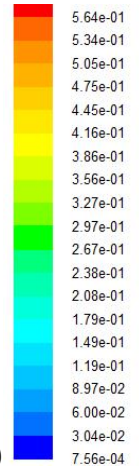

Figura 5 - Ângulo de repouso dinâmico para condição de rolamento (13 rpm) com 803,5 g: (a) Valor experimental $\left(\phi=50^{\circ}\right)$, (b) Valor simulado $\left(\phi=55^{\circ}\right) \operatorname{com} k=400, \eta=0.5, \mu_{f}=0.5$.

\section{CONCLUSÃO}

A abordagem CFD-DEM apresentou resultados satisfatórios para descrever o escoamento de grãos de fertilizantes em disco rotativo, desde que, os parâmetros do modelo sejam calibrados para que possam prever os regimes típicos deste equipamento. Também foi possível encontrar o ângulo de repouso dinâmico característico do regime de rolamento com boa concordância entre os resultados simulados e experimentais.

\section{REFERENCIAS}

BORTOLOTTI，C.T.; SANTOS，K.G.; FRANCISQUETTI，M.C.C.; DUARTE，C.R.; BARROZO, M.A.S. Hydrodynamic Study of a Mixture of West Indian Cherry Residue and Soybean Grains in a Spouted Bed. Can. J. Chem. Eng., v. 91, p. 871-1880, 2013.

CUNDALL, P.A.; STRACK, O.D.L. A discret numerical model for granular assemblies. Geotech., v. 29, p. 47-65, 1979.

DI RENZO, A.; DI MAIO, F.P. Comparison of contact-force models for the simulation of collisions in DEM-based granular flow codes. Chem. Eng. Sci., v. 59, p. 525-541, 2004.

LI, T.; GARG, R.; GALVIN, J.; PANNALA, S. Open-source MFIX-DEM software for gassolids flows: Part II - Validation studies, Powder Technol., v. 220, p. 138-150, 2012.

SILVERIO, B.C.; SANTOS, K.G.; DUARTE, C.R.; BARROZO, M.A.S. Effect of the friction, elastic and restitution coefficients on the fluid dynamics behavior of a rotary dryer operating with fertilizer. Ind. \& Eng. Chem. Res., v.53 p.8920-8926, 2014.

\section{AGRADECIMENTOS}

Os autores agradecem suporte do CNPq (460513/2014-1) e da Fapemig (APQ-01139-14). 